\title{
Comportamiento de la productividad en empresas del cluster plásticos establecidas en Bogotá D.C. dedicadas a la manufactura de envases, empaques y embalajes entre años 2003 a 2009
}

\author{
Andrés Giovanni Guarín Salinas* \\ Universidad de América
}

FECHA DE ENTREGA: 1 DE NOVIEMBRE DE 2013

FECHA DE EVALUACIÓN: 20 DE ENERO DE 2014

FECHA DE APROBACIÓN: 28 MARZO DE 2014

\begin{abstract}
Resumen El artículo hace una aproximación al estado de la productividad en el sector de plásticos en la ciudad de Bogotá, bajo los términos planteados en la función de producción de Cobb-Douglas, con el objetivo de establecer los puntos conexos entre un tipo de producto industrial específico y las posibles variaciones presentadas en relación al uso del capital, la fuerza de trabajo y la apropiación de tecnología. El documento está compuesto por cuatro partes, la primera de ellas, a manera de introducción, contextualiza el entorno bajo el cual opera la competitividad, vinculando dicho concepto con el sentido real que debe dársele a la productividad, resaltando su importancia para las organizaciones empresariales en la búsqueda de generar mayores beneficios. La segunda y tercera parte, respectivamente, describen el marco teórico y la metodología bajo la cual se fundamentó la investigación, particularmente la construcción del índice de Tornqvist-Theil y el análisis de la función de producción. Por último, se establecen las conclusiones a partir del análisis a dos modelos cuantitativos planteados.
\end{abstract}

\begin{abstract}
This article indicates the productivity status in the plastic cluster sector in the city of Bogotá, under the terms set by the production function of Cobb-Douglas in order to establish the related points between a specific industrial product and the possible variations presented regarding the use of capital, the labor force, and the appropriation of technology. This document has four parts: the first one contextualizes - as an introduction - the environment where competitiveness operates, by
\end{abstract}

* Artículo de investigación científica y tecnológica, que hace parte del conjunto de resultados del proyecto de estructuración de un modelo de competitividad para el clúster de plástico en la ciudad de Bogotá D.C., diseñado por el Centro de Investigación y Desarrollo Empresarial (COL0041544), bajo la línea de investigación en Lean Manufacturing, entre Marzo y Agosto de 2012. Más información en andres.guarin@profesores . uamerica. edu.co 
tying that concept to the real sense to be attributed to productivity. This way, we highlight its importance to the companies when looking to generate more benefits. The second and third parts describe the theoretical framework and the methodology used to base the research, respectively, emphasizing on the construction of the Tornqvist-Theil index and the analysis of the production function. Lastly, we established the conclusions after analyzing two quantitative models previously outlined.

Palabras Clave: cluster, competitividad, crecimiento, producción, productividad. JEL: C10, C020, C200, L590, L690.

Keywords: cluster, competitiveness, growth, production, productivity. JEL: C10, C020, C200, L590, L690.

\section{Introduccción}

\subsection{Marco conceptual}

A partir del momento en que Milton Friedman, al inicio de la década de los setenta, declaró como una especie de naturalis principia "que cualquier variación de la masa monetaria es seguida por una variación en el mismo sentido de los precios, de la producción y de los ingresos" [1]; se desencadenó un efecto creciente a escala mundial que en esencia consideraba el rol y la estructura del libre mercado más que suficiente, para asegurar la distribución óptima de los recursos y el uso efectivo de las capacidades de producción.

La corriente de pensamiento económico (con ciertos matices políticos reaccionaros), que se derivó de estos análisis, sentaron las bases de una ideología que redefiniría y dominaría de manera amplia, la visión capitalista del mundo durante el último cuarto del siglo XX y la primera década del siglo XXI; hasta el surgimiento en el año 2007 de la crisis económica mundial que afectó y puso contra las cuerdas a importantísimas instituciones financieras y que a partir de 2008 comprometió el nivel de empleo y los ingresos en nombre del pago de la deuda pública acaecida como consecuencia de los planes de rescate.

A pesar de lo anterior y de su comprobado desgaste histórico, la ideología neoliberal sigue profundamente arraigada en la mente de muchos analistas económicos y domina la agenda de muchos gobiernos; y aunque está en proceso de revisión, muchas de sus prácticas aún presentan vigencia debido entre muchos factores, al surgimiento en paralelo de un fenómeno que se encuentra enmarcado dentro de la interdependencia global actual; que lo hace concreto, visible y medible; y lo más relevante, que ha logrado sintetizar todo el conjunto de actividades humanas a un esquema de libre competencia, donde en teoría, los mejores prevalecerían siempre y cuando se mantengan en una constante dedicación, creatividad e inventiva, trayendo como consecuencia directa un "bienestar general".

El fenómeno descrito, es conocido como competitividad y ha logrado en el plazo de solo unas décadas impulsar una carrera desbocada para ocupar los primeros lugares dentro de un amplísimo conjunto de indicadores de todos los sabores y tendencias, establecidos principalmente por organizaciones multilaterales de 
las más disímiles características, las cuales construyen escalafones donde sitúan y posicionan a los diferentes países y por extensión, a las regiones, ciudades y organizaciones que hacen parte de su entramado social; en relación a su habilidad para ser más productivo y así generar proporcionalmente más riqueza que los competidores directos.

Colombia, no es ajena a este fenómeno y a partir de la década de los noventa hace su apropiación de los principios rectores del neoliberalismo, en cierta medida bajo el influjo de un relevante hecho histórico, como fue el de establecer un nuevo contrato social.

La Constitución que vio la luz en 1991, le hizo una gran apuesta a la modernidad y más aún a la postmodernidad [2], y fue escrita por los representantes que en su momento planteaban esas tesis y que en un afán de configurar un Estado más incluyente, crearon un grupo de derechos inalienables para todos los ciudadanos, que en la práctica resultaban muy costosos de implantar; por lo tanto se afirmó que el mercado debería actuar de una forma más directa y funcionar con menos regulación, sin llegar a tocar la distribución de la riqueza. El pacto social conformado entonces y que rige desde ese momento, no dejo establecido como aumentar en serio la productividad y como organizar la carga tributaria para lograr satisfacer los derechos de todos.

\subsection{Panorama de la Productividad}

Dentro del contexto anterior, se vislumbra que el camino más directo para alcanzar una mejor y sostenible calidad de vida, es mediante el aumento directo en los niveles de productividad de las empresas, que trae consigo una competitividad in crescendo que facilita el acceso a mercados; y por lo tanto a un aumento de las exportaciones, que se refleja en el aumento gradual de los ingresos per cápita de la población [3]. Un constante aumento en la productividad, coadyuva en el control a problemas estructurales de la economía, como la inflación, el desempleo, una balanza comercial deficitaria y una paridad monetaria inestable.

Colombia actualmente presenta bajos niveles de productividad y en promedio corresponde a un $20 \%$ de la productividad de un trabajador en los Estados Unidos; país con el cual se ha establecido un Tratado de Libre Comercio, desde Mayo 15 de 2012; lo que significa que se requieren de cinco trabajadores en Colombia para alcanzar el mismo nivel de producto que logra un trabajador norteamericano en una hora.

La principal causa para que se presente este nivel tan bajo en la productividad colombiana, tiene que ver con la baja eficiencia con la que se usan los recursos disponibles, la cual está estrechamente asociada a múltiples variables que explican el rezago en términos de competitividad, como son la informalidad empresarial, bloqueos para acceso a crédito, infraestructura y servicios logísticos precarios, poca innovación y desarrollo (traducido esto último en pocas patentes susceptibles de ser explotadas comercialmente). 


\subsection{Iniciativa frente a la competitividad}

La ciudad de Bogotá D.C. y su área metropolitana, es la conurbación más representativa que tiene Colombia en términos de crecimiento industrial y de potencial de negocios; solamente esta región hace un aporte al PIB por encima del $30 \%$ del total nacional y emplea cerca del $9,8 \%$ de toda la fuerza productiva del país; lo que la convierte en el foco de atención de muchos inversionistas y de muchas iniciativas que buscan hacerla más competitiva.

En esta región coexisten muchas instituciones de fomento al desarrollo económico, que buscan captar recursos locales, nacionales e internacionales, para el financiamiento de programas que permitan a diferentes sectores, mejorar las condiciones de operación técnica y hacerlos más atractivos a potenciales inversionistas.

En este sentido, sobresale una institución que por sus características se ha convertido de iure en un organismo consultivo de primer nivel y que en teoría tiene el propósito ulterior de aumentar la prosperidad de los habitantes de Bogotá, al ejecutar una propuesta de valor encaminada hacer fuerte la red de empresas que la componen y mejorar con esto el grado de competitividad de la región; esta organización es la Cámara de Comercio de Bogotá (CCB), la cual suscribió el 22 de Septiembre de 2008 con el Banco Interamericano de Desarrollo (BID), el convenio ATN/ME-11056, el cual dentro de su marco lógico de acción, tenía como fin, aumentar la competitividad y generar nuevas oportunidades de mercado para las PYME, con un aumento del $5 \%$ en su productividad.

La naturaleza de este convenio entró en absoluta concordancia con los lineamientos establecidos por la Comisión Regional de Competitividad (CRC) y sus ejes estratégicos transversales (estrategia V), entre los cuales se destaca el sectorial de apoyo a la transformación productiva y desarrollo de clústers, entre los que se encuentra el de plásticos (proyecto B-9), orientado principalmente al diseño y desarrollo de nuevos empaques, envases y embalajes que permitan alargar la vida útil y el transporte de los productos obtenidos en los sectores de alimentos, agroindustria y cosmética.

\subsection{Características del sector plásticos en Bogotá D.C.}

De la revisión de dos estudios técnicos, ver [4] y [5], que hacen una aproximación del sector de plásticos en Bogotá, se logra identificar en primera instancia que este sector es considerado aún una derivación de la industria petroquímica y por lo tanto presenta un alto nivel de dependencia, convirtiéndolo por reflejo, en uno de los de mayor dinamismo al ser arrastrado por el impulso reciente de la industria petrolera; permitiéndole desarrollar una incipiente tendencia exportadora, así como con una presencia importante de inversión extranjera.

Sin embargo al analizar el sector de manera más profunda, se encuentran algunas características vitales que deben ser atendidas para ganar competitividad, tanto en el ámbito nacional como en el entorno continental. Así, se identifica en primer lugar, que los niveles de calidad de los productos hechos en el clúster, son bajos, ya que las empresas (principalmente las PYMES), no cuentan con la 
tecnología y la infraestructura suficiente para producir bienes sofisticados que sean el resultado de procesos estructurados de innovación, que a su vez provengan de investigaciones estratégicas de nuevos mercados y de desarrollo estratégico de nuevos materiales, que permitan una usabilidad acorde con las exigencias actuales de impacto ambiental y de confort.

Las principales causas de la problemática arriba expuesta, corresponden en primera instancia, a la reducida gama de materias primas empleadas para poder manufacturar bienes con un alto valor agregado, en parte debido a la falta de una integración más fuerte con los proveedores de resinas plásticas; la segunda causa, es la imposibilidad de acceso a recursos de capital para invertir en tecnología, que incide de manera directa en el incremento de la calidad de los productos; la tercera causa, es la muy poca alianza entre empresas, centros de investigación e instituciones de fomento; que estén en capacidad de brindar el apoyo requerido, para hacer una sostenible reconversión industrial y una profunda capacitación de la fuerza laboral, en términos de mejoramiento continuo de los procesos de producción y el desarrollo de competencias laborales idóneas.

\section{Marco teórico}

El concepto de productividad, que esta implícitamente vinculado con el de competitividad, recibe una constante atención principalmente por parte de los productores [6], los cuales usan la información derivada de su medición, para lograr identificar una distinción entre los movimientos que representan las diferentes combinaciones de los factores productivos ${ }^{1}$ (independientemente de las proporciones que se usen), que permitan dar como resultado una cantidad determinada de producto, en respuesta a los cambios que surgen de las fluctuaciones de los precios relativos de estos mismos factores.

Así, si a un sector industrial le interesa determinar su productividad, debe estudiar los distintos niveles de producción, determinados en un mapa de múltiples posibilidades, que deben orientar las decisiones gerenciales hacia dos escenarios complementarios; el primero tiene que ver con la necesidad de establecer nuevas inversiones, que propicien un desarrollo gradual a nivel tecnológico; el segundo escenario, busca producir un efecto agregado de reducción de costos reales por medio de una reorganización empresarial, principalmente a nivel de los procesos industriales. El uso en conjunto de ambos escenarios, constituye un mecanismo para ir cerrando la brecha de conocimiento y de progreso, que incide de forma directa en el aumento de la competitividad [1].

La medida residual de la productividad de factores, también conocida como productividad total factorial (PTF), identificada como $A$, puede llegar a explicar hasta un tercio del crecimiento, de acuerdo a los trabajos realizados en este campo por el premio Nobel Robert Solow; donde su modelo de crecimiento exógeno, se explica y describe bajo la función de producción Cobb-Douglas (ecuación 1).

${ }^{1}$ Principalmente Capital y Trabajo, pero se consideran otros, como la adopción de tecnología, la desregulación de los mercados, la estabilidad jurídica, los derechos de propiedad intelectual, la disponibilidad de los insumos, consumo de energía, etc. 


$$
y=A_{\varphi}^{t} k^{\alpha}(h)^{1-\alpha} L
$$

Esta función de producción dado su homogeneidad, presenta un comportamiento multiplicativo importante, donde todas las variables que la componen, capital físico en planta y equipos $(k)$; fuerza de trabajo disponible $(h)$; el trabajo, representado en el número de horas puestas a disposición del sistema productivo $(L)$; al ser influenciadas por $A$ logran ofrecer rendimientos de escala constantes. Igualmente esta función tiene la propiedad de que $\alpha$ y $1-\alpha$, representan la participación del capital y de mano de obra, respectivamente.

De los dos enfoques existentes para medir la productividad de factores, se ha escogido para esta investigación, el que utiliza números índices, ya que representa la cuantificación de los productos finales manufacturados (outputs), en proporciones relativas al ingreso; en relación con los factores productivos (inputs) que se emplean para la generación de estos mismos productos, representados en proporciones relativas al costo.

De lo anterior, la productividad de factores, se establece como la razón de un índice de outputs $(Q)$, respecto a un índice de inputs $(X)$, en un periodo base $\varphi$ para un periodo de análisis $t$.

$$
A_{\varphi}^{t}=\frac{Q}{X}
$$

De los índices número más representativos, incluyendo el de Laspeyers y Paasche, para hacer la medición de la productividad de factores y que logra satisfacer todas las pruebas de inversión del tiempo, de precios y cantidades necesarias para no incurrir en sesgos estadísticos; está el de Tornqvist-Theil, el cual es un índice de cadena, que recoge los cambios estructurales ocurridos en un periodo de tiempo analizado; y requiere para su construcción, datos sobre cantidades de productos y sus factores, junto con sus correspondientes precios, que permitan ponderar el valor asignado a cada bien en la agregación.

El índice Tornqvist-Theil, es considerado un índice superlativo, lo que significa que puede llegar a ser exacto para funciones flexibles, al darles un tratamiento igual a los precios y a las cantidades, en todos los periodos. Las simetrías que establece este índice, proporcionan aproximaciones de segundo orden, cercanas a los índices que miden el costo de vida.

Por lo tanto, el índice de Tornqvist-Theil, en un tiempo $t$, para una serie de outputs, se puede calcular de acuerdo a los elementos planteados en la ecuación 3.

$$
P_{t}=\prod_{i=1}^{n}\left(\frac{p_{i, t}}{p_{i, \varphi}}\right)^{\frac{1}{2}\left[\frac{p_{i, \varphi} \cdot q_{i, \varphi}}{p_{\varphi} \cdot q_{\varphi}}+\frac{p_{i, t} \cdot q_{i, t}}{p_{t} \cdot q_{t}}\right]}
$$

Donde se requiere los datos de precios y cantidades de dos periodos de tiempo ( $\varphi$ y $t$ ), para cada uno de los $n$ artículos que se indexan a partir de $i=1$ hasta $i=n$; así, $p_{i, \varphi}$ y $q_{i, \varphi}$, es el precio y la cantidad, respectivamente, del artículo $i$ en el periodo de base $\varphi$; a su vez, $p_{i, t}$ y $q_{i, t}$, es el precio y la cantidad, respectivamente, del artículo $i$ en el periodo de análisis $t$; igualmente $p_{\varphi}$ y $q_{\varphi}$, 
son los preciosla tabla 4 y cantidades del periodo base; $p_{t}$ y $q_{t}$ representan los precios y cantidades del periodo de análisis.

Análogamente, el índice de Tornqvist-Theil, en un tiempo $t$, para una serie de inputs, se puede calcular mediante los elementos establecidos en la ecuación 4.

$$
C_{t}=\prod_{i=1}^{n}\left(\frac{c_{i, t}}{q_{i, \varphi}}\right)^{\frac{1}{2}\left[\frac{c_{i, \varphi} \cdot q_{i, \varphi}}{c_{\varphi} \cdot q_{\varphi}}+\frac{c_{i, t} \cdot q_{i, t}}{c_{t} \cdot q_{t}}\right]}
$$

Donde se requiere los datos de costos y cantidades de dos periodos de tiempo ( $\varphi$ y $t$ ), para cada uno de los $n$ artículos que se indexan a partir de $i=1$ hasta $i=n$; así, $c_{i, \varphi}$ y $q_{i, \varphi}$ es el costo y la cantidad, respectivamente, del artículo $i$ en el periodo de base $\varphi$; a su vez, $c_{i, t}$ y $q_{i, t}$ es el costo y la cantidad, respectivamente, del artículo $i$ en el periodo de análisis $t$; igualmente $c_{\varphi}$ y $q_{\varphi}$, son los precios y cantidades del periodo base; $c_{t}$ y $q_{t}$ representan los costos y cantidades del periodo de análisis.

\section{Metodología y análisis de resultados}

\subsection{Productividad de factores}

Para el análisis del comportamiento de la productividad en el clúster de plásticos en Bogotá D.C., se analizaron 26 tipos diferentes de producto, clasificados en 4 grupos, de acuerdo a las características de usabilidad en el segmento de empaques, envases y embalajes, según la codificación establecida por la Encuesta Anual Manufacturera (EAM), emitida por el Departamento Administrativo Nacional de Estadística (DANE), para los años 2002 al 2009, ver cuadro 1.

De la caracterización de los productos, se obtuvieron las cantidades producidas y su costo, al igual que el precio y las cantidades vendidas en los años referenciados.

Simultáneamente, se analizaron los tres principales procesos industriales ${ }^{2}$ de transformación del plástico (Extrusión, Inyección y Soplado), clasificados según los cambios de estado que sufre la resina a lo largo de la línea de fabricación para producir el tipo de producto final acorde a la codificación del DANE, para determinar el consumo de energía eléctrica (Kwh/Kg de producto), de los sistemas auxiliares hidráulicos, neumáticos, de refrigeración y de aire comprimido, que comprometen un nivel de avance tecnológico, donde se establece cierto progreso del proceso industrial frente al uso eficiente de la energía.

En este orden de ideas, el cálculo del número índice para outputs, teniendo como base el año 2002, al aplicar la ecuación 3, dio como resultado los valores expresados en el cuadro 2.

A lo anterior se añade, la medición del flujo de servicios derivados del trabajo, donde se asume una proporción fija del total de horas trabajadas, la cual refleja

\footnotetext{
${ }^{2}$ El plástico es moldeado a través de un conjunto de procesos primarios de tipo térmico, donde el material pasa de estado líquido y finalmente se solidifica. En procesos secundarios, como termoformado, doblado, laminado, sellado, cortado, impresión y peletizado entre otros se definen las características propias del producto final.
} 


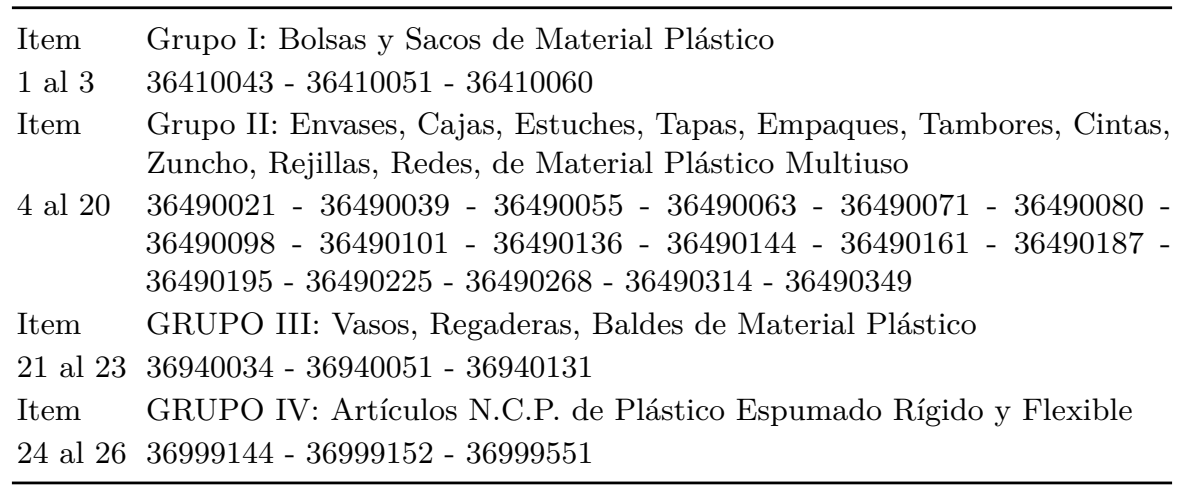

Cuadro 1. Codificación DANE para productos plásticos.

\begin{tabular}{cc}
\hline Año $(t)$ & $P_{t}$ \\
\hline 2003 & 1,120083285 \\
2004 & 1,191423397 \\
2005 & 1,243025838 \\
2006 & 1,256170454 \\
2007 & 1,291470414 \\
2008 & 1,328887939 \\
2009 & 0,930503732 \\
\hline
\end{tabular}

Cuadro 2. Índice para outputs.

la intensidad laboral a lo largo del proceso de fabricación, como una medida directa de la utilización de los recursos de producción. Así, el trabajo como factor de producción se descompone según la educación, sexo y ocupación; y son homologadas por la EAM en tres categorías: empleados administrativos, técnicos y obreros; cada uno de los componentes se pondero por su tasa de salario y de prestaciones sociales.

A su vez, los materiales intermedios que se consideraron como factor de producción, se dividieron en tres categorías: i) materias primas domésticas, ii) materias primas importadas y iii) costos indirectos de fabricación. En esta última categoría se incluyó los gastos de consumo en artículos complementarios como compra de repuestos y accesorios, trabajos de mantenimiento, subcontratación industrial, consumo de lubricantes y combustibles. Los anteriores elementos fueron ponderados para inferir su valor, por lo tanto esta variable se define como las compras netas de inventarios de materias primas más los costos indirectos de fabricación. En este orden de ideas, el cálculo del número índice para Inputs, 
teniendo como base el año 2002, al aplicar la ecuación 4, dio como resultado los valores expresados en el cuadro 3.

\begin{tabular}{cc}
\hline Año $(t)$ & $C_{t}$ \\
\hline 2003 & 1,12705501 \\
2004 & 1,19124266 \\
2005 & 1,26770137 \\
2006 & 1,25620501 \\
2007 & 1,31922910 \\
2008 & 1,32962125 \\
2009 & 0,93739623 \\
\hline
\end{tabular}

Cuadro 3. Índice para outputs.

En concordancia con los datos anteriores, el cálculo de la productividad de factores, una vez determinados los índices de outputs e inputs ${ }^{3}$ respectivamente, mediante la ecuación 2, arrojó los resultados expresados en en el cuadro 4.

\begin{tabular}{cc}
\hline Año $(t)$ & $A_{\varphi}^{t}$ \\
\hline 2003 & 0,99381421 \\
2004 & 1,00015172 \\
2005 & 0,98053522 \\
2006 & 0,99997249 \\
2007 & 0,97895841 \\
2008 & 0,99944848 \\
2009 & 0,99264719 \\
\hline
\end{tabular}

Cuadro 4. Productividad de factores.

Una $A<1$, indica que la productividad para ese periodo en particular, representó un uso deficiente de los recursos de producción, por lo tanto se está perdiendo competitividad.

En contraste, cuando $A>1$, en un periodo en particular, se está haciendo un uso eficiente de los recursos de producción, que permite ir ganando competitividad.

\footnotetext{
${ }^{3}$ Los índices tanto de outputs como de inputs, cumplen con las propiedades de carácter matemático para este tipo de medida estadística.
} 
Cuando $A=1$, significa que existe una coincidencia tanto en el periodo base como en el de análisis, que refleja una variación nula, por lo tanto se mantiene estático.

Al llevar los resultados a un plano de coordenadas (Figura 1), se puede inferir que $A$ para el sector de plásticos en Bogotá D.C., presenta una oscilación entre 0.9789 y 1.0001 , la cual es una franja débil en términos de productividad, donde se puede deducir que este sector industrial, a pesar de su reconocido potencial, es poco dinámico, dado a mantener su participación en el mercado, con pocas variaciones en el uso de los recursos de producción y con productos poco diferenciados que no comprometen el uso de nuevas tecnologías.

Al analizar la medición de $A$ con otro tipo de factores [7], como son el de Prosperidad Económica (FPE) y el de Concentración del Empleo (FCE), el clúster de estudio puede definirse como uno que solamente logra abastecer el mercado local y se encuentra aún lejos de llegar a establecerse en comercios internacionales de amplias exigencias técnicas y normativas.

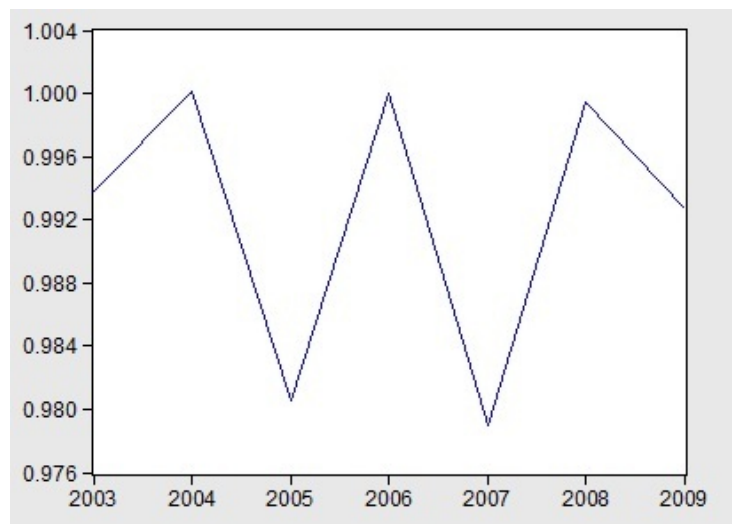

Figura 1. Serie de productividad de factores $A$.

Igualmente cabe resaltar, que ante la inspección a la figura 1 , de la serie de $A$, se refleja la presencia de tres ciclos de negocios, caracterizados por la alternancia entre fases de expansión (valle a pico), donde se presenta un incremento de la actividad económica agregada; y de contracción (pico a valle), donde por el contrario se presenta una reducción significativa de la actividad económica.

En el flujo constante entre picos y valles de la figura 1, se identifican cuatro puntos de quiebre, de los cuales se resalta uno en particular, que hace parte de la cronología de estudio, y es el periodo comprendido entre los años 2007 a 2009, ya que en esta franja de tiempo se evidencia el inicio de un ciclo de expansión, que es constatado mediante el análisis del Factor de Dependencia del Clúster (FDC) $[7]$. 
De la observación anterior se puede establecer un marco de referencia para el seguimiento de los factores de producción en años posteriores de acuerdo a la tendencia que se insinúa.

\subsection{Función de producción}

Dentro del análisis econométrico llevado a cabo durante la investigación, se establecieron dos modelos a partir de la linealización de la función de producción, es decir, aplicando logaritmos a las variables originales de la ecuación 1. Así, en el primer modelo, expresado según los términos de la ecuación 5 , la función de producción toma las características de una función monótona creciente en sus variables, $k$ capital, $L$ trabajo y $h$ fuerza laboral disponible; la función expresada de esta forma, indica que el producto puede llegar a incrementarse por tres razones fundamentales; primero, que las personas trabajen más (un mayor nivel de $L$ ), segundo, porque tienen más equipo para trabajar (una $k$ más elevada), o tercero, porque el capital y el trabajo se utilizan en la producción de forma más eficiente (un nivel de $A$ más elevado).

$$
\log y=\beta_{0}+\beta_{1} \log k+\beta_{2} \log h+\beta_{3} \log L+\mu
$$

Al sistematizar los datos, se muestra a la función de producción bajo la estimación de la ecuación con los coeficientes sustituidos (Figura 2).

$$
\begin{gathered}
L Y=C(1)+C(2) \cdot L K+C(3) \cdot L H+C(4) \cdot L L \\
L Y=0.2066+3.5787 \cdot L K-0.6539 \cdot L H+0.1594 \cdot L L
\end{gathered}
$$

\begin{tabular}{|c|c|c|c|c|}
\hline \multicolumn{5}{|c|}{$\begin{array}{l}\text { Dependent Variable: LY } \\
\text { Method: Least Squares } \\
\text { Date: } 06 / 26 / 12 \text { Time: } 14: 53 \\
\text { Sample: } 20032009 \\
\text { Included observations: } 7\end{array}$} \\
\hline Variable & Coefficient & Std. Error & t-Statistic & Prob. \\
\hline $\mathrm{C}$ & 0.206614 & 2.003500 & 0.103127 & 0.9244 \\
\hline LK & 3.578762 & 1.741687 & 2.054767 & 0.1322 \\
\hline $\mathrm{LH}$ & -0.653999 & 1.284936 & -0.508974 & 0.6458 \\
\hline LL & 0.159405 & 0.369885 & 0.430959 & 0.6956 \\
\hline R-squared & 0.599945 & \multicolumn{2}{|c|}{ Mean dependent var } & -2.678092 \\
\hline Adjusted R-squared & 0.199890 & \multicolumn{2}{|c|}{ S.D. dependent var } & 0.131773 \\
\hline S.E. of regression & 0.117869 & \multicolumn{2}{|c|}{ Akaike info criterion } & -1.142925 \\
\hline Sum squared resid & 0.041679 & \multicolumn{2}{|c|}{ Schwarz criterion } & -1.173833 \\
\hline Log likelihood & 8.000237 & \multicolumn{2}{|c|}{ Hannan-Quinn criter. } & -1.524948 \\
\hline F-statistic & 1.499656 & \multicolumn{2}{|c|}{ Durbin-Watson stat } & 1.212454 \\
\hline Prob(F-statistic) & 0.373599 & & & \\
\hline
\end{tabular}

Figura 2. Coeficientes para el modelo 1.

El segundo modelo propuesto para el estudio, toma la expresión de la ecuación 8 ; donde la función de producción se plantea en términos de la relación del 
producto por unidad de mano de obra, estableciendo que sólo es posible obtener algo de producto usando una mínima cantidad de trabajo $L$, dado el supuesto que la función de producción es homogénea en grado 1.

$$
\log \frac{y}{L}=\beta_{0}+\beta_{1} \log k+\beta_{2} \log h+\mu
$$

Lo anterior conlleva a establecer que las productividades marginales de las variables son positivas al igual que decrecientes, donde por cada unidad adicional de una de ellas, el beneficio generado disminuye al aumentar la cantidad de dicha variable.

Al sistematizar los datos, se muestra a la función de producción bajo la estimación de la ecuación con los coeficientes sustituidos (Figura 3).

$$
\begin{gathered}
L Y S O B R E L=C(1)+C(2) \cdot L K+C(3) \cdot L H \\
L Y S O B R E L=1.4463+4.3522 \cdot L K-0.0699276 \cdot L H
\end{gathered}
$$

\begin{tabular}{|c|c|c|c|c|}
\hline \multicolumn{5}{|c|}{$\begin{array}{l}\text { Dependent Variable: LYSOBREL } \\
\text { Method: Least Squares } \\
\text { Date: } 06 / 26 / 12 \text { Time: } 14: 57 \\
\text { Sample: } 20032009 \\
\text { Included observations: } 7\end{array}$} \\
\hline Variable & Coefficient & Std. Error & t-Statistic & Prob. \\
\hline C & 1.446338 & 2.754236 & 0.525132 & 0.6273 \\
\hline LK & 4.352277 & 2.440355 & 1.783460 & 0.1491 \\
\hline LH & -0.069928 & 1.798681 & -0.038877 & 0.9709 \\
\hline R-squared & 0.443045 & \multicolumn{2}{|c|}{ Mean dependent var } & -2.682614 \\
\hline Adjusted R-squared & 0.164568 & \multicolumn{2}{|c|}{ S.D. dependent var } & 0.184240 \\
\hline S.E. of regression & 0.168399 & \multicolumn{2}{|c|}{ Akaike info criterion } & -0.427439 \\
\hline Sum squared resid & 0.113432 & \multirow{2}{*}{\multicolumn{2}{|c|}{$\begin{array}{l}\text { Schwarz criterion } \\
\text { Hannan-Quinn criter. }\end{array}$}} & -0.450621 \\
\hline Log likelihood & 4.496037 & & & -0.713957 \\
\hline F-statistic & 1.590957 & \multicolumn{2}{|c|}{ Durbin-Watson stat } & 1.825474 \\
\hline Prob(F-statistic) & 0.310198 & & & \\
\hline
\end{tabular}

Figura 3. Coeficientes para modelo 2.

Del análisis de resultados de los dos modelos, se deja entre ver la presencia de dos hechos importantes, que se incluyen por razones de coherencia y porque parecen indicar el rumbo que debe tomar la investigación a futuro. El primer hecho, es que aunque el nivel de los coeficientes es relativamente bajo, lo que hace entender que no existe un ajuste acorde entre las variables, se presupone que esto no excluye que existan otras relaciones funcionales entre las mismas; como es el caso del impacto de la acumulación de capital humano, en términos del grado de educación de la fuerza de trabajo y el desarrollo de competencias que establezcan un conjunto de destrezas laborales propias para los procesos de transformación del plástico. 
Para este acercamiento en primer lugar se propone una tasa de inversión de capital humano que proporciona una conexión entre los costos y el crecimiento educativo; en segundo lugar, todavía hay retornos constantes a los tres factores $(k, L$ y $h)$, y retornos decrecientes a los dos factores reproductivos $(k \mathrm{y} h)$.

El segundo hecho, tiene relación con que el $p$-valor asociado a los dos modelos, no representa una significación estadística suficiente para un $95 \%$ de confianza, lo cual indica que es muy infrecuente obtener una muestra como la actual; por lo tanto las bases de datos consultadas dado su volumen y calidad de información, no permiten conclusiones más robustas y solamente orientan el proceso hacia un nivel de diagnóstico que aunque hace parte de la descripción del horizonte de eventos no alcanza a ser totalmente concluyente.

Sin embargo es posible que mediante la variación de los supuestos realizados, se incluyan más consideraciones, relacionadas a otros tipos de factores que potencialmente puedan limitar el incremento de la productividad, como pueden ser el tamaño y la madurez de las organizaciones para afrontar procesos de mejoramiento continuo.

\section{Conclusiones}

Los resultados obtenidos muestran la importancia de establecer una medición no paramétrica de la productividad de factores, que permita la aproximación al grado de competitividad del sector industrial del plástico en Bogotá D.C. en términos asociados a la naturaleza del progreso tecnológico del mismo.

Los hallazgos marcan una tendencia del comportamiento del clúster de estudio en relación al uso eficiente de los recursos, donde se identifica una franja muy débil donde fluctúan los índices calculados, que en promedio para los años 2003 al 2009 se encuentra en 0,99 lo que indica que el sector es poco dinámico en su gestión, quizás como consecuencia de su fuerte vínculo con la industria petroquímica, que no lo deja liderar una profundización tecnológica acorde con las expectativas del mercado, que logre una modernización de los procesos de transformación para producir bienes sofisticados.

Las bases de datos consultadas para los cálculos de los factores de producción, particularmente el de fuerza laboral disponible $(h)$, arrojaron resultados que distan de los esperados, ya que no reflejan en su totalidad la incidencia de otros fenómenos que pueden llegar a definir la productividad en el sector de plásticos; por lo tanto es necesario refinar la medición con la incorporación de elementos complementarios que permitan la estimación de una convergencia condicional, donde el crecimiento del clúster este asociado directamente con la inclusión de variables de capital humano, que le hagan tomar distancia de su actual situación de estado estacionario. 


\section{Agradecimientos}

A la economista y profesora de la Universidad Nacional y Universidad Javeriana, Maria Esperanza Cuenca, por su interés en el tema y orientación frente a los resultados obtenidos de los dos modelos cuantitativos planteados.

Igualmente a los ingenieros investigadores, Jorge Alberto Medina Perilla y Jairo Ernesto Perilla Perilla, de la Universidad de los Andes y de la Universidad Nacional, respectivamente, por la inmersión en los procesos de transformación del plástico.

Al Departamento Nacional de Estadística (DANE), especialmente a la Dirección de Metodología y Producción Estadística y a su director, Eduardo Efraín Freire, por el sumistro y ayuda en el manejo de los datos necesarios para esta investigación.

\section{Referencias}

1. Toussaint, E.: Un coup d'oeil dans le rétroviseur, Lieja (Bélgica), (2010).

2. Gómez, H.: Revista Malpensante website. [en línea]. Disponible: http://www . elmalpensante.com (2011)

3. Dirección de Estudios e Investigaciones: Informe Nacional de Competitividad, Consejo Privado de Competitividad, Bogotá, (2007).

4. Banco Interamericano de Desarrollo y Cámara de Comercio de Bogotá: Guía metodológica para el uso eficiente de la energía en el sector plástico, Proyecto OPEN, (2008).

5. Califa, C., Peralta, L.: Estudio prospectivo, estratégico y tecnológico del clúster de plásticos en Bogotá, Tesis de Ingeniería, Universidad de América, Bogotá D.C., Abril 2012, S.T. T658. 5C128.

6. Bain, D.: The Productivity Prescription: The Manager's Guide to Improving Productivity and Profits, New York (USA), ed., McGraw-Hill, (1982).

7. Zuniga, C.: Texto básico de economía agrícola: su importancia para el desarrollo local sostenible, Universidad Nacional Autónoma de Nicaragua, León, (2011).

8. Guarín, A.: Cálculo de los factores de concentración del empleo, de prosperidad económica y de dependencia del clúster de plásticos en Bogotá D.C., Revista de Investigación Universidad de América, 1 ed. vol 5, pp.52-66, Enero - Junio (2012).

9. Díaz, A., Sáenz, J.: Productividad total factorial y el crecimiento económico de México. Economía y Desarrollo, Revista Universidad Autónoma de Colombia, 1 ed. vol 1, pp. 105-180, (2002).

10. Alfonso, V., Arango, L., Arias F., Pulido, J.: Ciclos de negocios en Colombia: 1980-2010, en Borradores de Economía (651), Banco de la República, 2011, pp. 1-10. (2011).

11. Fundación de Investigaciones Económicas, Productividad, Competitividad, Empresas: Los engranajes del crecimiento, Ed. FIEL, Buenos Aires, (2002).

12. Mojica, F.: La construcción del futuro, 1ed., Universidad Externado de Colombia Convenio Andrés Bello, Bogotá, (2005). 